\title{
INVESTIGATION OF 3D CULTURE OF HUMAN ADIPOSE TISSUE-DERIVED MESENCHYMAL STEM CELLS IN A MICROFLUIDIC PLATFORM
}

\author{
Ceren ÖZEL ${ }^{1,3}$ (D), Yücel KOÇ ${ }^{2,3}$ (iD), Ahmet Emin TOPAL ${ }^{3}$ (D), Aliakbar EBRAHIMI ${ }^{3,7}$ (iD \\ Tayfun ŞENGEL ${ }^{1,6}$ (D) , Hamed GHORBANPOOR ${ }^{3,4}$ (i) , Fatma DOĞAN GÜZEL ${ }^{5}$ (iD), \\ Onur UYSAL 1, 3 (iD), Ayla EKER SARIBOYACI 1,3 id, Hüseyin AVCI 1, 3, 7, 8 * id \\ ${ }^{1}$ Department of Stem Cell, Institute of Health Sciences, Eskişehir Osmangazi University, Eskişehir, Turkey \\ ${ }^{2}$ Department of Chemical Engineering, Eskişehir Osmangazi University, Eskişehir, Turkey \\ ${ }^{3}$ Cellular Therapy and Stem Cell Production Application and Research Center (ESTEM), Eskişehir Osmangazi University, \\ Eskişehir, Turkey \\ ${ }^{4}$ Department of Biomedical Engineering, Eskișehir Osmangazi University, Eskișehir, Turkey \\ 5 Department of Biomedical Engineering, Ankara Yildirim Beyazıt University, Ankara, Turkey \\ ${ }^{6}$ Central Research Laboratory Research and Application Center (ARUM), Eskişehir Osmangazi University, Eskişehir, \\ Turkey \\ ${ }^{7}$ Department of Metallurgical and Materials Engineering, Eskişehir Osmangazi University Eskişehir, Turkey. \\ ${ }^{8}$ Translational Medicine Research and Clinical Center (TATUM), Eskişehir Osmangazi University, Eskişehir, Turkey
}

\begin{abstract}
Mesenchymal stem cells (MSCs) are multipotent stem cells that can support various tissues including bone marrow, adipose tissue, and synovial fluids, from which they can be readily isolated. The objective of this study is to harness the advantages of microfluidic systems for controlling and enhancing the maintenance and viability, and regenerative properties of MSCs by providing a 3D culture microenvironment with gelatin methacrylate (GelMA) hydrogel and exposing the cells to a slow fluid flow and low shear stress conditions. GelMA has methacryloyl groups and can be crosslinked by a photocuring process using biocompatible photoinitiators. The most common used photoinitiator for cellular encapsulation within hydrogels is the ultraviolet (UV) initiator 2-hydroxy-4'-(2-hydroxyethoxy)-2-methylpropiophenone (Irgacure 2959 or I2959), but due to its low water solubility and the necessity of using a shorter wavelength light $(365 \mathrm{~nm})$, it can lead to cellular phototoxic and genotoxic effects. To overcome these limitations, lithium phenyl-2,4,6-trimethylbenzoylphosphinate (LAP) have recently been used with GelMA as an alternative photoinitiator. Because LAP is highly water soluble and has a 10 times faster polymerization rate, and it requires a visible light $(\lambda=405 \mathrm{~nm})$ which makes it much safer for the cells, we use $10 \%$ GelMA together with $0.05 \%$ LAP photoinitiator for bioprinting human adipose tissue derived MSCs (hAT-MSCs) onto a membrane that has a $40 \mu \mathrm{m}$ mesh size. To demonstrate a microfluidic culture advancement for improving the biological activities and regenerative capacity of the cells including cell adhesion, growth, viability and proliferation capacity as ultimate goals of this study, the membrane carrying the bioprinted construct was placed in a PDMS microchannel and exposed to the fluid to obtain dynamic microenvironments found in the human body. As a result, the cells were successfully maintained in the microfluidic 3D cell culture for two days, with a high cell viability of $99 \%$.
\end{abstract}

Keywords: MSCs, Organ on a chip, GelMA, 3D cell culture, Cell adhesion

\section{INTRODUCTION}

Organ-on-a-chip (also called organ chip or OoC) is a miniaturized bioreactor technology in a microfluidic cell culture device which reconstitutes the physiology and architecture of a notable functional unit of a specific organ of interest [1-6]. Organ chip technology is a cost-effective approach for the study of disease and treatment and has a potential for predicting drug-induced organ-specific responses and reducing the use of animal experimentation. Organoids are three-dimensional cell culture models composed of either natural extracellular matrix (ECM) molecules or biomaterials interacting with organ-specific cells that self-organize into their peculiar physiology and anatomy and mimic an

*Corresponding Author: havci@ogu.edu.tr

Received: 18.08.2021 Published: 30.11.2021 
associated organ in vivo [2,7-9]. Organoids typically contain stem cells, though not necessarily, as they can be comprised of some primary cells or differentiated cells. Biomimetic platforms of many organs including the lung, liver, small intestine, kidney, and some other tissues like bone marrow and bloodbrain barrier can be designed and studied as organ chips [1]. Studies of drug development and disease, and cosmetic pipelines will likely involve organ-on-a-chip technology soon.

Mesenchymal stem cells, or also called mesenchymal stromal cells (MSCs) are multipotent stem cells that can be isolated from many tissues/organs including bone marrow, adipose tissue, dental pulp, and amniotic or synovial fluids [10]. MSCs are unique cells for regenerative medicine that can support functions of many tissues and organs by releasing soluble factors such as growth factors, anti-apoptotic and anti-inflammation factors, and they can synthesize extracellular matrix (ECM) mainly composed of collagen fibrils, a key component of tissues [11]. Stem cell differentiation is known to be governed by a variety of soluble factors including growth factors, hormones and cytokines whereas numerous studies performed in recent decades point out that in addition to chemical factors, mechanical cues can also have remarkable roles on cellular differentiation, proliferation, biomechanical properties and tissue development. Likewise, MSCs have been shown to be highly responsive to extracellular mechanical cues [12-15], including responses to different magnitudes of shear stresses [16,17]. For instance, Riddle et al. uses parallel plate flow chambers to test the effects of high shear stress on human MSCs and report that oscillatory fluid flow-induced shear stress with high flow rates (i.e. $1-50 \mathrm{ml} / \mathrm{min}$ flow rates) resulted with increased cell proliferation due to a high shear stress-induced increase in intracellular calcium concentration [18]. On the contrary, high magnitude shears stresses above $10 \mathrm{mPa}$ levels can exert damage on rat MSCs and lead to cell detachments and reduced proliferation [19]. Slow fluid flow and low shear stresses at flow rates ranging from few microliters up to hundreds of microliters per min upregulate osteogenic differentiation and proliferation of MSCs in microfluidic devices [20-22]. As such, shear stresses and interstitial fluid flow can influence and regulate the cellular behavior of MSCs. Furthermore, by using microfluidic devices, how interstitial fluid flow influences the MSC viability and differentiation in the presence of 3D cultures can be investigated in microchannels as well.

Photo-crosslinking is a chemical crosslinking method performed by photoinitiation followed by photocuring in bioprinting, and photocurable biomaterials such as gelatin-methacrylate (GelMA) are frequently used as bio-ink material in a bioprinting process. To induce chemical cross-linking of bioink right after the printing process, the construct is exposed to light (UV or visible light) in a way that stiffens enough but does not cause any major damage on the cells [23]. 2-hydroxy-4'-(2hydroxyethoxy)-2-methylpropiophenone (Irgacure 2959), as a most common UV photoinitiator used for cellular encapsulation within hydrogels, has low water solubility, and needs a shorter wavelength of UV light $(365 \mathrm{~nm})$ that can cause cellular phototoxic and genotoxic effects in cells. However, recent studies show that lithium phenyl-2,4,6-trimethylbenzoylphosphinate (LAP), as an alternative photoinitiator, can be used to overcome Irgacure limitations. LAP has high water solubility and a 10 times faster polymerization rate than Irgacure. Also, in comparison with Irgacure, LAP needs a higher wavelength of visible light $(\lambda=405 \mathrm{~nm})$ for photoinitiation, that is much safer for cells [24-26]. GelMA has been used together with MSCs in recent bioprinting studies whereby tissue regeneration ended up with providing enhanced regenerative properties such as high cell viability, and improved cell adhesion and proliferation [27]. In a recent report, human bone marrow-derived MSC-laden GelMA constructs ended up having larger MSC spreading area and a high cell viability of 90\% [28]. In the literature, only a few studies like Miri et al. investigate GelMA encapsulated MSCs as a bio-ink in microfluidic culture setups and organ chip studies [29]. As such, MSCs seeded in a 3D matrix of GelMA have not been thoroughly investigated in microchannels under, for instance, fluid flow with low shear stress conditions.

In this study, human adipose tissue-derived mesenchymal stem cells (hAT-MSCs) encapsulated with photo-cross-linkable GelMA hydrogel were used as bio-ink to fabricate a construct for performing 3D 
cell culture in a PDMS-based microfluidic channel. The GelMA/hAT-MSCs construct was bioprinted on a membrane which was later cut into a small piece with an appropriate size before transferring from Petri dish to the microfluidic device. The cells were cultured under low shear stress conditions for two days and the viability of the cells was determined by live/dead assay.

\section{EXPERIMENTAL}

\subsection{Production of the Microfluidic Chip}

The microfluidic (MF) chip template was prepared using a 3D printer. SLA/DLP 3D printing technology (micraft 100) was used to fabricate of mold with resolution 30-35 micron and velocity $30 \mathrm{sec}$ per layer. OOkuma FP60 resin was applied to prepare the mold. The mold inside area was $39 \times 46 \times 5 \mathrm{~mm}$. Then, PDMS (Sylgard 184 silicon elastomer base and Sylgard 184 silicon elastomer curing agent, Dow Corning, USA) layer was prepared using the soft lithography method [30]. In this study we used the low molecular weight Sylgard ${ }^{\mathrm{TM}} 184$ PDMS polymer (Component A: $\mathrm{Mn}=5.8 \times 10^{3} \mathrm{Da}$; Component B: $\left.\mathrm{Mn}=7.5 \times 10^{3} \mathrm{Da}\right)$ [31]. For this, $5.85 \mathrm{~g}(1.00 \mathrm{mmol})$ of PDMS (component A) and $0.65 \mathrm{~g}(0.087 \mathrm{mmol})$ of crosslinker (Component B) was used for preparation of upper and bottom PDMS microchannels. The mixture of PDMS and crosslinker (9:1) was prepared by stirring for up to $3 \mathrm{~min}$ and added to molds and vacuumed in the desiccator for $20 \mathrm{~min}[32,33]$. It was then left to stand at $50{ }^{\circ} \mathrm{C}$ overnight, and then the microfluidic chip was prepared by peeling off the PDMS layer. On a PDMS layer, there is a microchannel and the input with output of the channel (top layer). The length of the channels is $15 \mathrm{~cm}$, the width is $1000 \mu \mathrm{m}$ and the height of the channel is $1000 \mu \mathrm{m}$. The other PDMS layer consists of a flat surface without a microchannel (bottom layer). A porous polyethylene terephthalate (PET) membrane with $40 \mu \mathrm{m}$ pore size (pluriStrainer ${ }^{\circledR}$ pluriSelect, Leipzig, Germany) was used as a membrane. Cells were bioprinted onto the membrane which was later placed between two PDMS layers. Finally, the chip was sandwiched between poly (methyl methacrylate) (PMMA) frames and fastened by screws. Figure 1 shows a schematic view and a final version image of the microfluidic device.

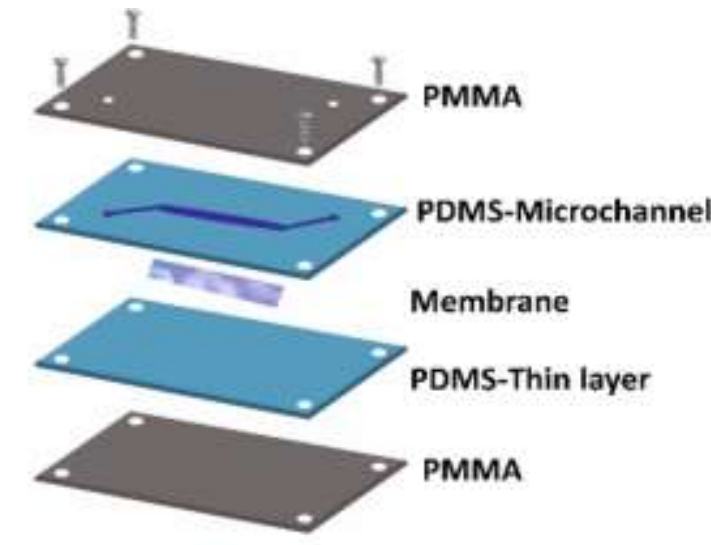

(a)

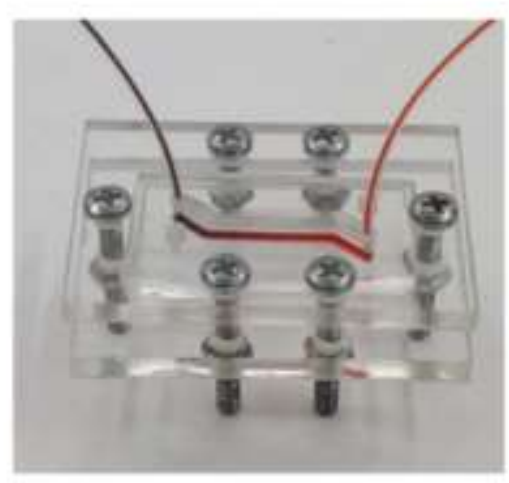

(b)

Figure 1. (a) Schematic view and (b) final version image of microfluidic cell culture device. 


\subsection{Isolation, Culture, and Characterization of MSCs}

Human adipose tissue-derived mesenchymal stem cells (hAT-MSCs) were isolated by surgical removal from healthy donors undergoing surgical operation. Tissue samples were harvested from the informed/consented healthy patients under Eskişehir Osmangazi University Clinical Research Ethics Committee permission (Decision number: 23/Date 30.05.2019). Tissue samples were washed several times with Hanks' balanced salt solution (HBSS) with 5\% penicillin-streptomycin and without calcium and magnesium. After the washing process, tissues were minced into small blocks and a single cell suspension of adipose tissue cells was obtained by using enzymatic digestion (0.075\% type I collagenase, Sigma), and mechanically with shaker water bath $37^{\circ} \mathrm{C}$ for approximately $60 \mathrm{~min}$. The cell suspensions were filtered using a $70 \mu \mathrm{m}$ cell strainer to separate individual cells from debris and undigested adipose tissue fragments. hAT-MSCs were cultured and characterized as in previously described protocols depending in vitro differentiation experiments and immunophenotyping by flow cytometry [34].

\subsection{On-chip Experiments}

MSCs were mixed with $10 \%$ (w/v) gelatin methacrylate (GelMA) and $0.05 \%$ (w/v) lithium phenyl2,4,6-trimethylbenzoylphosphinate (LAP) photoinitiator (CELLINK, Sweden). This prepolymer/MSCs blend was bioprinted by micropipette extrusion on the membrane before the chip was assembled. Due to its biocompatibility, GelMA was selected as hydrogel biomaterial to be used in the microfluidic chip [35]. The cells encapsulated within GelMA bioprinted on the membrane and then statically cultured in a 6-well polystyrene plate for 2 days in a cell culture incubator (Panasonic). After cell proliferation was observed on GelMA at the end of day 2, the membrane coated with GelMA/MSCs was integrated into the microfluidic chip as shown in Figure 1a and 2f, and the cells were grown in another incubator (Esco Lifesciences Group) for 2 days under the dynamic culture conditions. Syringe pump (NE-4000, New Era Pump Systems Inc., NY) was used with a flow rate of $0.5 \mu 1 / \mathrm{min}$ to inject the culture medium through Tygon tubing into the microchip placed in the incubator. Waste culture medium was collected from the outlet of microchip in a $15 \mathrm{ml}$ falcon tube inside the incubator. $37{ }^{\circ} \mathrm{C}$ with $5 \%$ $\mathrm{CO}_{2}$ gas supply and humid incubator conditions were used for all the experiments. Figure 2 shows the steps applied for on-chip experiments.

Shear stress is the stress created when a tangential force is acting on hAT-MSCs by laminar flow of culture media over area of cell contact with the surface of PDMS microchannel. Shear rate and shear stress can be determined by equations 1 and 2 [36].

$$
\begin{aligned}
& \text { Shear Rate }(\gamma)=\frac{Q}{W h^{2} 10^{4}} \\
& \text { Shear Stress }(\tau)=\text { Shear Rate }(\gamma) \times \operatorname{Viscosity}(\mu)
\end{aligned}
$$

where $W$ is the width and $h$ the height of the channel, $\mu$ is viscosity of culture media, and $Q$ is the volumetric flow rate used in the microfluidic culture setup. 


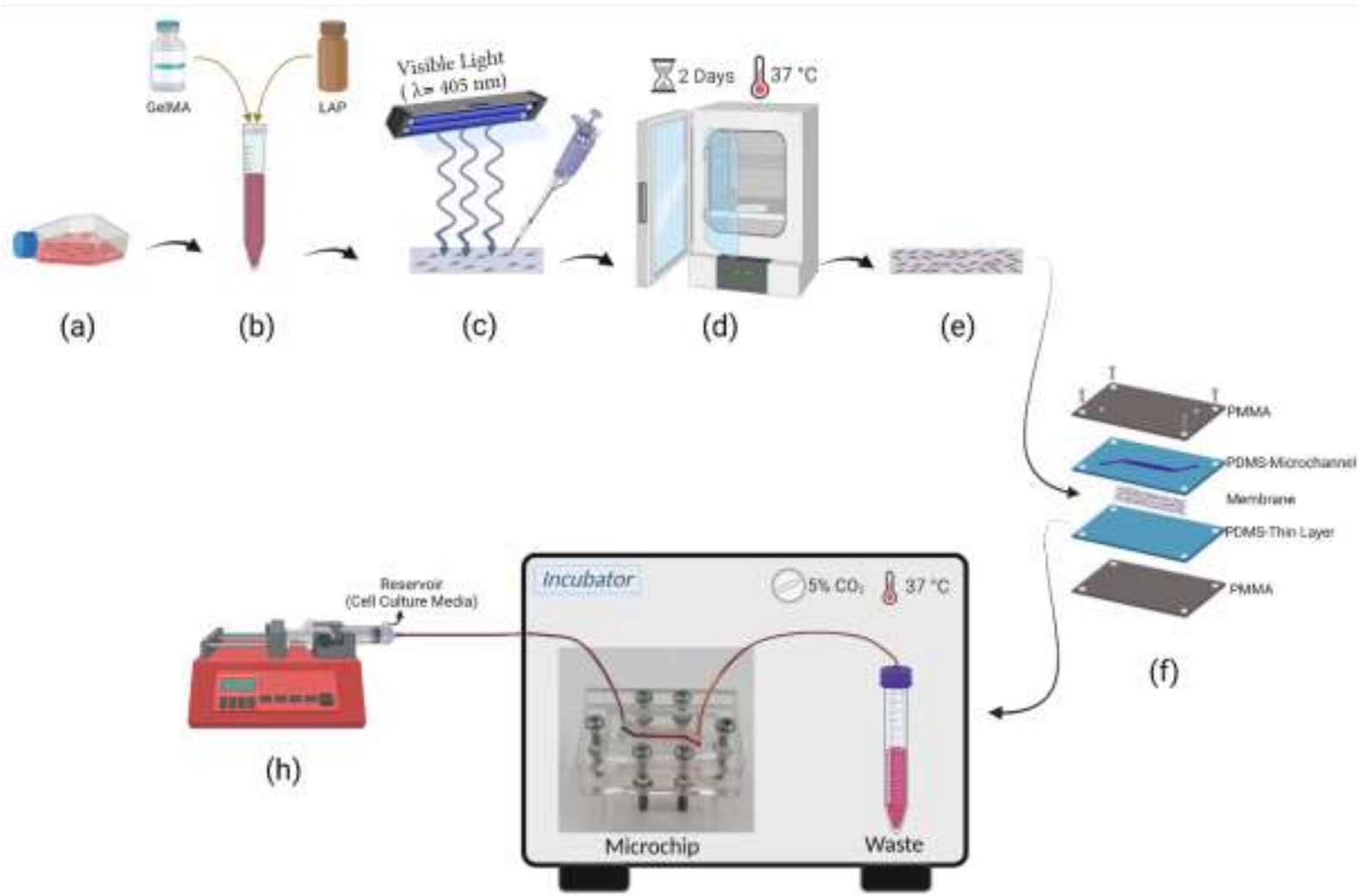

(g)

Figure 2. Schematic view of microfluidic cell culture experiment that includes (a) trypsinization, (b) mixing $10 \%$ (w/v) GelMA with MSCs, (c) GelMA/MSCs seeding on the membrane followed by photocuring (405 $\mathrm{nm})$, (d) incubation of the coated membrane in a Petri dish for 2 days at $37{ }^{\circ} \mathrm{C}$ with $5 \% \mathrm{CO}_{2}$, (e) observation of cell growth, (f) schematic representative of the microfluidic culture device, (g) image of the final version microfluidic culture device shown inside the incubator and $(\mathrm{h})$ a syringe attached on a syringe pump placed outside the incubator.

\subsection{Live/Dead Assay}

Cells were imaged with a live/dead assay to verify cell viability. To assess the viability of cultured MSCs cells within the microfluidic chip, the cells were incubated in Live/Dead (AAT Bioquest ${ }^{\circledR}$ ) solution for 30 min in dark and imaged using Carl ZEISS AXIO OBSERVER D1 fluorescence microscope. The percentage of cell viability was calculated by dividing the number of live cells (green) by the total number of cells, as shown in the equation below:

Percentage of Live Cells $=($ Live Cells/Total Cell Number $) \times 100$

\section{RESULTS AND DISCUSSION}

Stem cells are unique cells known for their self-renewal properties that ensure the preservation of the stem cell pool, while they can divide and differentiate into several cell type of tissues within the body according to their differentiation potential. This phenomenon refers to the potency (i.e. stem cells can be totipotent, pluripotent, multipotent, oligopotent or unipotent) [37]. MSCs are multi-potent cells that can differentiate into multiple types of cells present in specific organs. In stem cell research, it is important to maintain stem cells in undifferentiated state or controlled differentiation. The main reason for that is because stem cells are highly responsive to microenvironment alterations such as mechanical stimuli and biochemical signals that can change stem cell fate, and eventually leading to loss of potency 
and stem cell identity [38]. Microfluidic technology has important potential for stem cell research. By utilizing a sophisticated microfluidic environment design, manipulation of stem cells can be controlled better than in conventional cell culture techniques. To direct the stem cell differentiation, it is crucial to know the role of various biochemical cues (e.g., soluble factors including trophic factors like growth factors and hormones, and also glucose, oxygen concentrations, etc.) in the decision-making process. Microfluidic devices have been used to study microenvironment mainly by focusing on the following two aspects: screening a wide range of conditions in a high-throughput fashion and reconstructing the physiological environment like heterogeneous, complex and 3D growth conditions. By utilizing the advantages of nanotechnology like micro- and nanofabrication tools and using various biomaterials that are biocompatible and bear hydrogel properties and/nanofibrous structures and assemblies which cells like to attach and live with, many different works have been performed using stem cells in microfluidic devices containing different polymeric materials with well-defined geometries and patterns [22].

In this study, we aim to provide in vivo like $3 \mathrm{D}$ and microfluidic culture conditions for the maintenance human adipose derived-mesenchymal stem cell in vitro. MSCs were obtained from liposuction materials of healthy donors and cultured conventional method before using in a microfluidic bioreactor device. Afterwards, the cells were subjected separately to both 3D microfluidic culture and static control which had the same 3D culture conditions used in for encapsulation of MSCs with GelMA hydrogel and culture on a membrane. In this regard, the isolated cells from human adipose tissue adhered on the culture flasks and were successfully cultured, and as a result, most cells displayed a spindle-shaped and fibroblast-like morphology, as shown in Figure 3. The cultured cells reached a monolayer confluency in the primary culture 7 days after the first passage and were expanded until they reached passage 3 (P3). Morphology of the cells indicate that the isolated cells can attach and expand on the surface of polystyrene cell culture flasks and proliferate over subcultures.

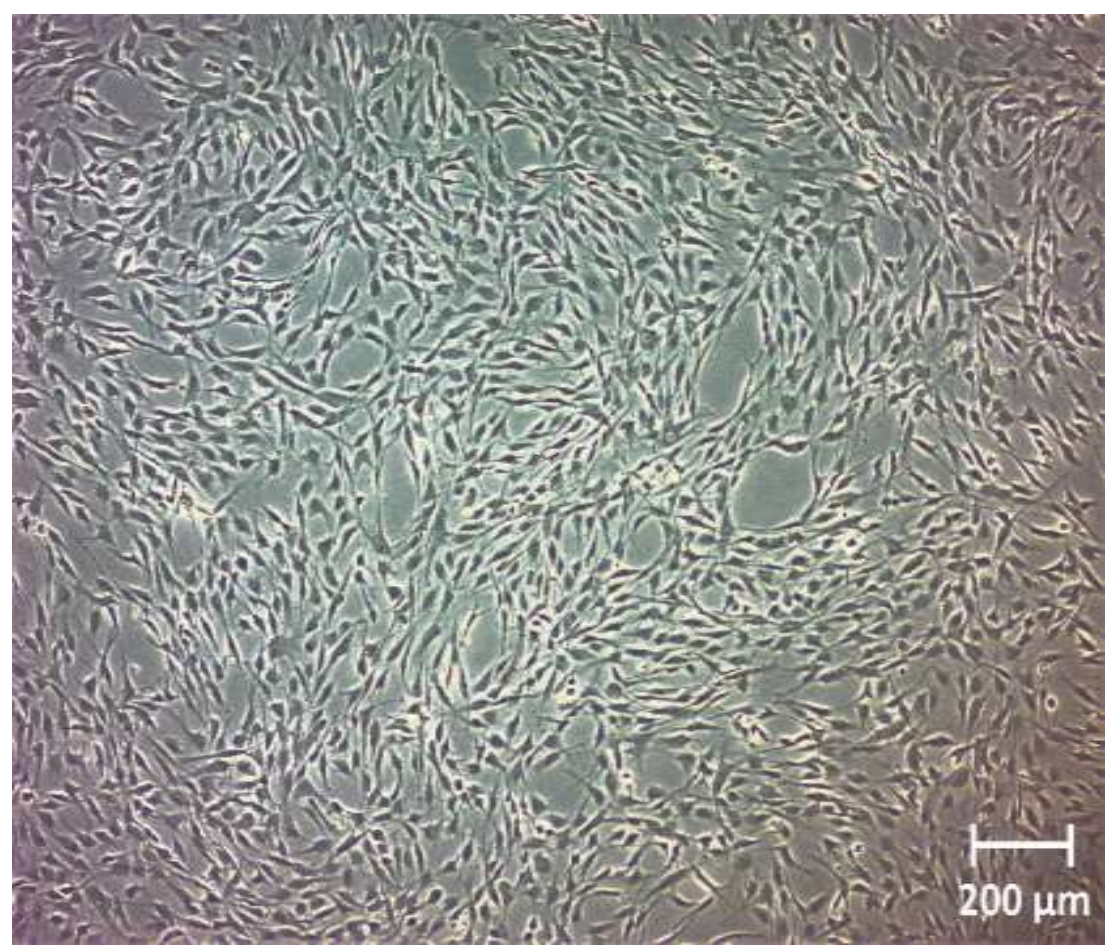

Figure 3. Phase contrast microscopy image of hAT-MSCs in a polystyrene culture dish showing the MSC morphology of the expanded cells (scale bar $200 \mu \mathrm{m}$ ). 
The cells isolated from human adipose tissue adhered to polystyrene flasks (Figure 3), and flow cytometry was used to show that they were phenotypically positive for CD90, CD73, CD29, and CD105 markers but negative for CD34, CD45, MHC class II (HLA-DR) and hematopoietic lineage markers as shown in Figure 4. These results suggest that the isolated cells can be identified as MSCs, as previously described [39]. Moreover, previous works by our group showed that these cells could differentiate into chondrocytes, adipocytes, and osteoblasts [34, 40, 41].
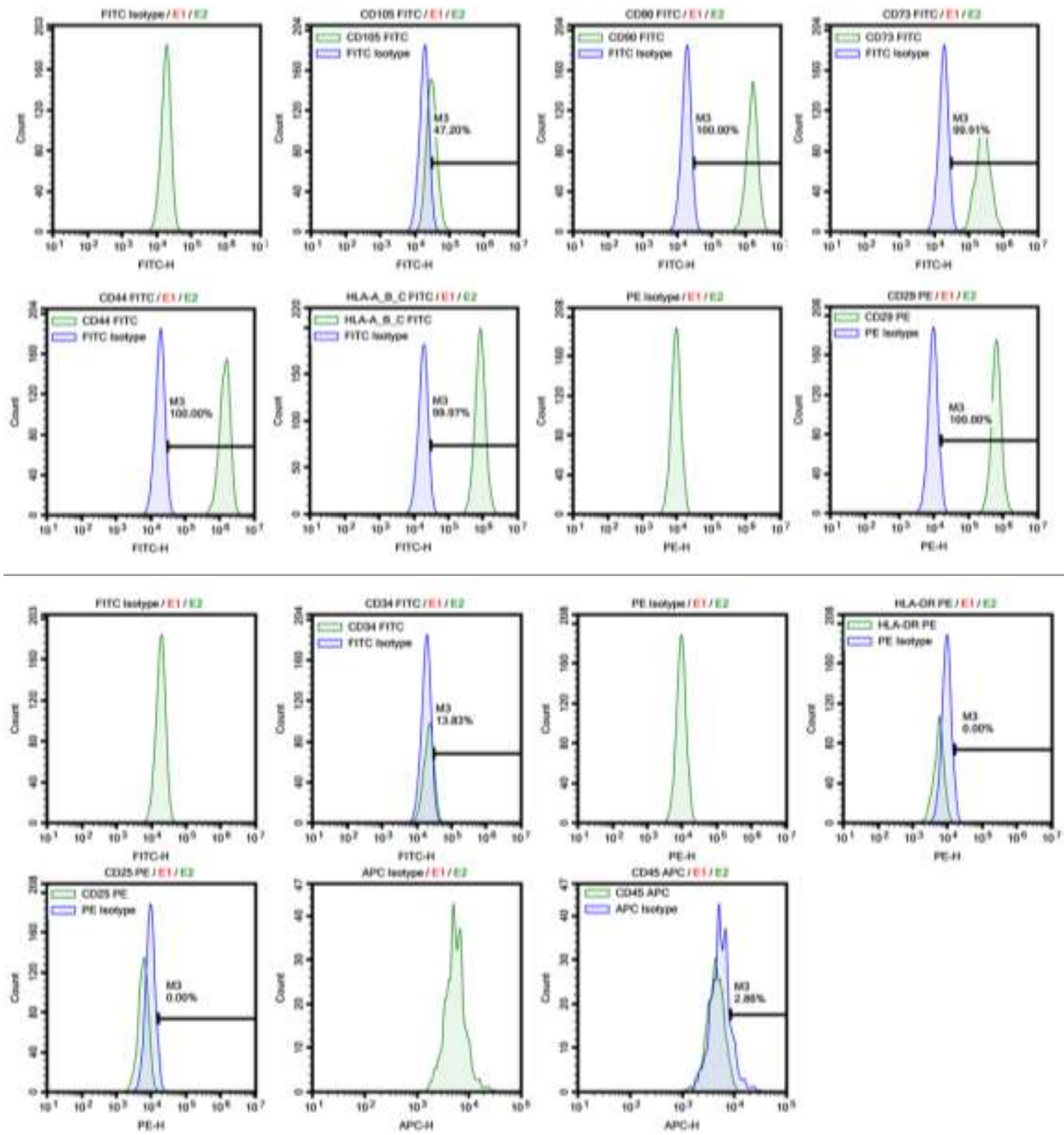

Figure 4. The immunophenotypic characterization of MSCs isolated from human adipose tissue by flow cytometry. Flow cytometry analysis of MSCs were performed at P3 (P: passage number).

MSCs were stained with calcein AM (green) within the microfluidic chip for live cells and with propidium iodide (red) for dead cells, which was followed by observing at an inverted fluorescence microscope (Zeiss Axio Observer D1), as shown in Figure 5. The microfluidic chip culture system 
containing the hAT-MSCs could be maintained successfully for 2 days with approximately $99 \%$ of cell viability within $10 \%$ (w/v) GelMA. Fibroblast-like and spindle-shaped morphologies of MSCs were observed on microfluidic chip culture (Fig. 3 and 5b). On the other hand, the morphology and cytoskeletal structure of the MSCs in static culture conditions were slightly more filamentous. Cell adhesion is key to formation of morphological characteristics of cells. Thus, ECM components such as collagen and its derivates including gelatin-based hydrogels such as GelMA can regulate cytoskeletal rearrangements, cell traction forces and shape $[42,43]$. Here, the GelMA/hAT-MSCs construct was bioprinted on a PET membrane with $40 \mu \mathrm{m}$ pore size of which we considered a membrane for allowing the cells and GelMA to attach on it, and this approach could be used in future organ-on-a-chip studies. As a future experiment, cell adhesion molecules can be immunostained, cell-cell contact interactions and cell-ECM interactions between hAT-MSCs and GelMA can also be analyzed in detail. However, imaging of the MSCs directly inside the microfluidic device comes with difficulty of resolving the details of cytoskeletal structures of the cells, especially when using a typical inverted fluorescence microscope.

(a)

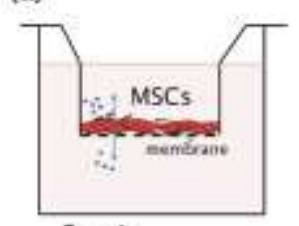

Static

culture

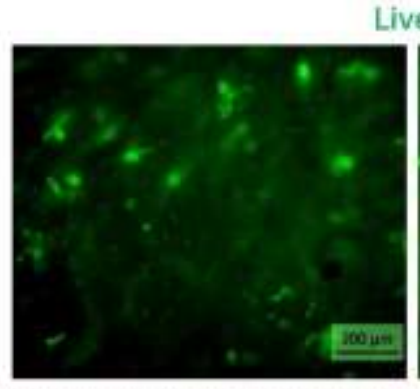

Live

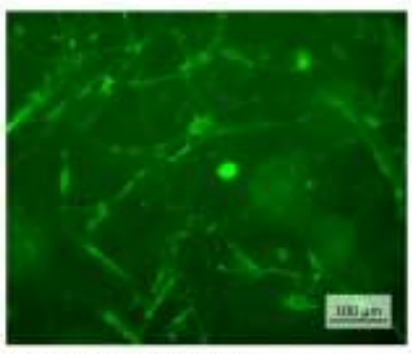

(b)

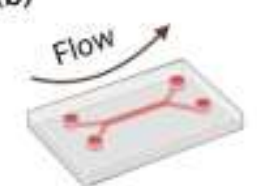

Microfluidic culture
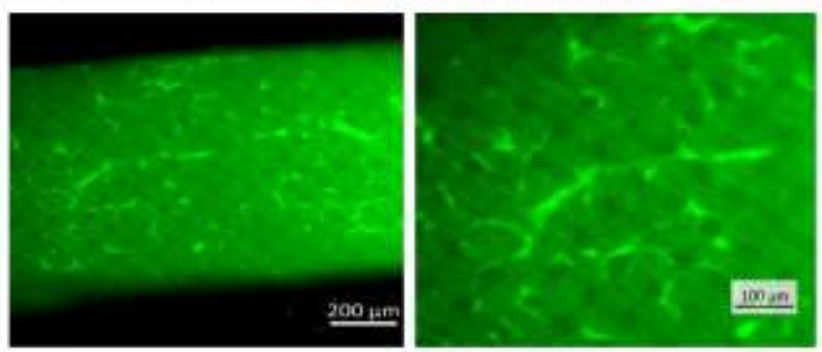
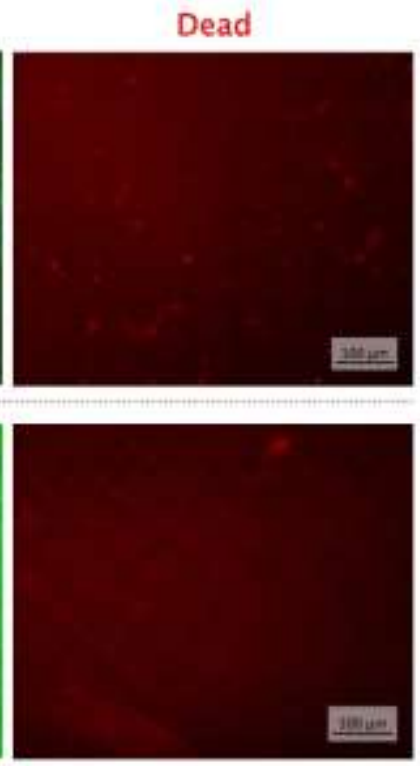

Figure 5. Live/dead assay images of static culture of MSCs on membrane under fluorescence microscope captured at the end of day 2 (a). Live/dead assay images of microfluidic chip culture MSCs on membrane under fluorescence microscope captured at the end of day 2 (b). The green mesh-like background view shows the membrane, and also microfluidic channel area of the chip on b. Scale bar of the images on the left is $200 \mu \mathrm{m}$, and scale bars of the images at the middle and on the right are $100 \mu \mathrm{m}$.

In an organism, stem cells reside in a specific anatomical location, that is called a stem cell niche, that consists of biochemical cues (e.g., cytokines, proteins, growth factors, glucose, oxygen, or inorganic components like calcium, etc.), stromal and other tissue-related cells (in some cases immune cells), extracellular matrix (ECM) of which GelMA was used for mimicking in this study. Blood vessels have a regulatory role in stem cell niche, and MSCs can be found surrounding capillaries to differentiate or control other cells in response to injury or tissue homeostasis. In order to mimic vascular environment, we used a microfluidic channel and cell culture medium (DMEM) instead of blood flow.

GelMA is known to support cell attachment and viability and promote cellular differentiation of many cell types including MSCs [29,44]. Moreover, GelMA has also been reported to form well-defined bioprinted constructs that contain MSCs in addition to allowing cell viability and maintenance [29]. Here, we use a GelMA/hAT-MSCs construct bioprinted on a membrane via micropipette extrusion method followed by photocuring and then tested it in a PDMS microfluidic channel. An important aspect 
of our study is that GelMA provides a physiological relevance as it consists of gelatin, that is, a baked mixture of collagenous proteins and also provides a mesh structure as an extracellular cue that the cells can respond to and like to attach. As a result of this study, the viability of the cells on GelMA in a 3D cell culture system was preserved under a fluid flow with low shear stress conditions. Moving from a static culture of GelMA-entrapped MSCs on the membrane $(40 \mu \mathrm{m})$ to its corresponding dynamic culture setup in a microchannel under slow fluid flow (i.e., with a flow rate of $0.5 \mu \mathrm{l} / \mathrm{min}$ ) does not reduce the viability of the cells and also does not cause a dramatic change in the morphology and appearance of the cells. This result may suggest that the GelMA-entrapped hAT-MSCs under slow fluid flow and hence exposed to low shear stress can be a representative of in vivo conditions, and the 3D microenvironment provided to the cells here can be used to imitate a stem cell niche present in human body. Stem cells have the ability to self-renew and can stay in a quiescent state in the body and can also become active and start differentiating upon receiving a bioactive signal or a significant change in the environment. Mechanical cues can play significant roles on mesenchymal stem cell decisions to proliferate or differentiate, for example, stem cells residing on a soft matrix at subkiloPascal levels can become rounder and stay quiescent whereas an increase in the stiffness of the matrix or substrate they attach towards kiloPascal levels or more can influence the stem cell fate as human MSCs have been reported to have a tendency to choose a stiffer substrate/matrix rather than a compliant one [45].

Here we calculate the shear stress value acting on hAT-MSCs used in this study as follows: by assuming that the viscosity $\mu$ of the culture medium is $0.89 \mathrm{mPa} \cdot \mathrm{s}$ (DMEM) as previously reported for water at room temperature and the properties have been shown to be almost identical to those for water [46]. We calculate the shear rate as $0.05 \mathbf{s}^{-1}$ by using equation 1 , and the magnitude of shear stress acting on hAT-MSCs as $0.0445 \mathrm{mPa}$ from equation 2, as also presented in Table 1.

Table 1. The flow rate and shear stress profiles to achieve the desired shear rate and shear stress within the microchannel.

\begin{tabular}{|c|c|c|c|}
\hline Shear Rate $(\boldsymbol{\gamma})$ & Shear Stress $(\boldsymbol{\tau})$ & Volumetric Flow Rate $(\boldsymbol{Q})$ & Viscosity $(\boldsymbol{\mu})$ \\
\hline $0.05 \mathrm{~s}^{-1}$ & $0.0445 \mathrm{mPa}$ & $0.5 \mu \mathrm{l} / \mathrm{min}$ & $0.89 \mathrm{mPa} \cdot \mathrm{s}$ \\
\hline
\end{tabular}

Flow-induced shear stresses that MSCs can respond to by proliferation and osteogenesis were previously reported as $10^{-4} \mathrm{~Pa}$ and $10^{-5} \mathrm{~Pa}$, respectively, where bone-marrow derived human MSCs and PET matrices were used [16-17]. As the shear stress acting on hAT-MSCs we report in this study is between those two values, our results imply that hAT-MSCs used in this study are expected to show proliferative properties, as supported by the viability result obtained in the microfluidic culture experiments shown in Figure 5. In addition, BrdU staining, or another similar assay should be done in order to further investigate and show that they can also have such good proliferative profiles in this microfluidic setup. High shear stresses, such as flow induced-deviatoric shear stresses, it is reasonable to say that very high stresses acting on cells under fast fluid flows at $\mathrm{ml}$ per min levels or greater can be detrimental on MSCs and cause cell detachments due to convection [17], however, values converging to 0.01-0.1 Pa interval as to play remarkably important roles in developmental biology such as mesenchymal condensation and tissue morphogenesis [47-50].

\section{CONCLUSIONS}

In conclusion, we showed that the hAT-MSCs could be successfully cultured with a high viability by using our microfluidic chip design under fluid flow conditions with low shear stress. Therefore, this microfluidic bioreactor culture has the potential for maintaining hAT-MSCs and improving their stem cell characteristics for further studies including self-renewal, differentiation potential and senescence phenotype. Furthermore, this setup can be tested as a candidate setup to be used in organ-on-a-chip studies inasmuch as GelMA is considered a biocompatible material which mimics the extracellular microenvironment of MSCs inside human body. Microfluidic devices are powerful tools for the 
reconstruction of stem cell microenvironment and recapitulation of important physiological functions for both high-throughput detection of biological processes and controlling the stem cell fate within a 3D microenvironment resembling the physiological conditions. As a future study, biomarker expressions of MSCs can be determined via immunocytochemistry at the end of microfluidic chip culture. The cells can be evaluated with their senescence phenotype and the expression of MSC-specific genes related with self-renewal and differentiation.

\section{ACKNOWLEDGMENTS}

This study was carried out as a part of thesis of Ceren ÖZEL within the scope of 100/2000 The Council of Higher Education (CoHE) Doctoral Scholarship program-spring term of the 2017-2018 academic year in the field of biomaterials and tissue engineering. This study was also supported by Turkish Scientific and Technological Council (TÜBİTAK 1004-Regenerative and Restorative Medicine Research and Applications) under the grant numbers of 20AG003 and 20AG031.

\section{CONFLICT OF INTEREST}

The authors stated that there are no conflicts of interest regarding the publication of this article.

\section{REFERENCES}

[1] Ingber DE. Developmentally Inspired Human 'Organs on Chips'. Development, 2018; 145(16):dev156125.

[2] Bhatia SN, Ingber DE. Microfluidic Organs-on-Chips. Nat Biotechnol, 2014; 32(8):760-72.

[3] Avci H, Güzel FD, Erol S, Akpek A. Recent Advances in Organ-on-A-Chip Technologies and future Challenges: A Review. Turkish Journal of Chemistry, 2017; 42(3):587-610.

[4] Zhang YS, Aleman J, Shin SR. et al. Multisensor-Integrated Organs-on-Chips Platform for Automated and Continual in Situ Monitoring of Organoid Behaviors. Proceedings of the National Academy of Sciences, 2017; 114(12):E2293-E2302.

[5] Shin SR, Zhang YS, Kim D-J. et al. Aptamer-Based Microfluidic Electrochemical Biosensor for monitoring Cell-Secreted Trace Cardiac Biomarkers. Analytical Chemistry, 2016; 88(20):10019-10027.

[6] Shin SR, Kilic T, Zhang YS. et al. Label- Free and Regenerative Electrochemical Microfluidic Biosensors for Continual Monitoring of Cell Secretomes. Advanced Science, 2017; 4(5):1600522.

[7] Mancio-Silva L, Fleming HE, Miller AB. et al. Improving Drug Discovery by Nucleic Acid Delivery in Engineered Human Microlivers. Cell Metabolism, 2019; 29(3):727-735. e3.

[8] de Souza N. Organoids. Nature Methods, 2018; 15(1):23-23.

[9] Garbioglu DB, Demir N, Ozel C, Avci H, Dincer M. Determination of Therapeutic Agents Efficiencies of Microsatellite Instability High Colon Cancer Cells in Post- Metastatic Liver Biochip Modeling. The FASEB Journal, 2021; 35(9):e21834. 
[10] Uccelli A, Moretta L, Pistoia V. Mesenchymal Stem Cells in Health and Disease. Nature Reviews Immunology, 2008; 8(9):726-736.

[11] Pers YM, Ruiz M, Noël D, Jorgensen C. Mesenchymal Stem Cells for the Management of Inflammation in Osteoarthritis: State of The Art and Perspectives. Osteoarthritis and Cartilage, 2015; 23(11):2027-2035.

[12] Topal AE. Mesenchymal Stem Cell Mechanics on Osteoinductive Peptide Nanofibers. PhD, Bilkent University, Ankara, Turkey, 2017.

[13] Topal AE, Tansik G, Ozkan AD, Guler MO, Dana A, Tekinay AB. Nanomechanical Characterization of Osteogenic Differentiation of Mesenchymal Stem Cells on Bioactive Peptide Nanofiber Hydrogels. Advanced Materials Interfaces, 2017; 4(20):1700090.

[14] Engler AJ, Sen S, Sweeney HL, Discher DE. Matrix Elasticity Directs Stem Cell Lineage Specification. Cell, 2006; 126(4):677-689.

[15] Mousavi SJ, Hamdy Doweidar M. Role of Mechanical Cues in cell Differentiation and Proliferation: a 3D Numerical Model. PloS one, 2015; 10(5):e0124529.

[16] Zhao F, Chella R, Ma T. Effects of Shear Stress on 3- D human Mesenchymal Stem Cell Construct Development in a Perfusion Bioreactor System: Experiments and Hydrodynamic Modeling. Biotechnology and Bioengineering, 2007; 96(3):584-595.

[17] Higuera GA, van Boxtel A, van Blitterswijk CA, Moroni L. The Physics of Tissue Formation with Mesenchymal Stem Cells. Trends in Biotechnology, 2012; 30(11):583-590.

[18] Riddle RC, Taylor AF, Genetos DC, Donahue HJ. MAP Kinase and Calcium Signaling Mediate Fluid Flow-Induced Human Mesenchymal Stem Cell Proliferation. American Journal of Physiology-Cell Physiology, 2006; 290(3):C776-C784.

[19] Dash SK, Sharma V, Verma RS, Das SK. Low Intermittent Flow Promotes Rat Mesenchymal Stem Cell Differentiation in Logarithmic Fluid Shear Device. Biomicrofluidics 2020; 14(5):054107.

[20] Kim KM, Choi YJ, Hwang J-H. et al. Shear Stress Induced by An Interstitial Level of Slow Flow Increases the Osteogenic Differentiation of Mesenchymal Stem Cells Through TAZ Activation. PloS one, 2014; 9(3):e92427.

[21] Bissoyi A, Bit A, Singh BK, Singh AK, Patra PK. Enhanced Cryopreservation of MSCs in Microfluidic Bioreactor by Regulated Shear Flow. Scientific Reports, 2016; 6(1):1-13.

[22] Zhang J, Wei X, Zeng R, Xu F, Li X. Stem Cell Culture and Differentiation in Microfluidic Devices Toward Organ-on-A-Chip. Future Science OA, 2017; 3(2):FSO187.

[23] Costa- Almeida R, Domingues RM, Fallahi A. et al. Cell- Laden Composite Suture Threads for Repairing Damaged Tendons. Journal of Tissue Engineering and Regenerative Medicine, 2018; 12(4):1039-1048. 
[24] Lim KS, Galarraga JH, Cui X, Lindberg GC, Burdick JA, Woodfield TB. Fundamentals and Applications of Photo-Cross-Linking In Bioprinting. Chemical Reviews, 2020; 120(19):1066210694.

[25] Wong DY, Ranganath T, Kasko AM. Low-Dose, Long-Wave UV Light Does Not Affect Gene Expression of Human Mesenchymal Stem Cells. PloS one, 2015; 10(9):e0139307.

[26] Lawrence KP, Douki T, Sarkany RP, Acker S, Herzog B, Young AR. The UV/Visible Radiation Boundary Region (385-405 nm) Damages Skin Cells and Induces "dark" Cyclobutane Pyrimidine Dimers in Human Skin in vivo. Scientific Reports, 2018; 8(1):1-12.

[27] Rajabi N, Rezaei A, Kharaziha M. et al. Recent advances on bioprinted gelatin methacrylatebased hydrogels for tissue repair. Tissue Engineering Part A. 2021; 27(11-12):679-702.

[28] Yin J, Yan M, Wang Y, Fu J, Suo H. 3D Bioprinting of low-Concentration Cell-Laden Gelatin Methacrylate (GelMA) Bioinks with A Two-Step Cross-Linking Strategy. ACS Applied Materials \& Interfaces, 2018; 10(8):6849-6857.

[29] Miri AK, Nieto D, Iglesias L. et al. Microfluidics- Enabled Multimaterial Maskless Stereolithographic Bioprinting. Advanced Materials, 2018; 30(27):1800242.

[30] Didarian R, Ebrahimi A, Ghorbanpoor H, Dizaji AN, Hashempour H, Guzel FD, Avci H. Investigation of Polar and Nonpolar Cyclotides Separation from Violet Extract Through Microfluidic Chip. 8. International Fiber and Polymer Research Symposium; 18-19 June 2021, Eskişehir Osmangazi University, Eskisehir, Turkey.

[31] Gupta NS, Lee K-S, Labouriau AJP. Tuning Thermal and Mechanical Properties of Polydimethylsiloxane with Carbon Fibers. Polymers, 2021; 13(7):1141.

[32] Dizaji AN, Ozturk Y, Ghorbanpoor H. et al. Investigation of the Effect of Channel Structure and Flow Rate on On-Chip Bacterial Lysis. IEEE Trans Nanobioscience, 2021; 20(1):86-91.

[33] Kaur J, Ghorbanpoor H, Öztürk Y. et al. On- Chip Label- Free Impedance- Based Detection of Antibiotic Permeation. IET Nanobiotechnology, 2021; 15(1):100-106.

[34] Ozdemir AT, Ozgul Ozdemir RB, Kirmaz C. et al. The Paracrine Immunomodulatory Interactions Between the Human Dental Pulp Derived Mesenchymal Stem Cells and CD4 T Cell Subsets. Cell Immunol, 2016; 310:108-115.

[35] Gao G, Schilling AF, Hubbell K. et al. Improved Properties of Bone and Cartilage Tissue from 3D Inkjet-Bioprinted Human Mesenchymal Stem Cells by Simultaneous Deposition and Photocrosslinking in PEG-GelMA. Biotechnology Letters, 2015; 37(11):2349-2355.

[36] Dupuy A, Ju LA, Passam FH. Straight Channel Microfluidic Chips for the Study of Platelet Adhesion under Flow. Bio-protocol, 2019; 9(6):e3195.

[37] Zakrzewski W, Dobrzyński M, Szymonowicz M, Rybak Z. Stem Cells: Past, Present, and Future. Stem Cell Research \& Therapy, 2019; 10(1):1-22. 
[38] Park D, Lim J, Park JY, Lee S-H. Concise Review: Stem Cell Microenvironment on A Chip: Current Technologies for Tissue Engineering and Stem Cell Biology. Stem Cells Translational Medicine, 2015; 4(11):1352-1368.

[39] Dominici M, Le Blanc K, Mueller I. et al. Minimal Criteria for Defining Multipotent Mesenchymal Stromal Cells. The International Society for Cellular Therapy Position Statement Cytotherapy, 2006; 8(4):315-7.

[40] Karaöz E, Demircan PÇ, Erman G, Güngörürler E, Sarıboyacı AE. Comparative Analyses of Immunosuppressive Characteristics of Bone-Marrow, Wharton's Jelly, and Adipose TissueDerived Human Mesenchymal Stem Cells. Turkish Journal of Hematology, 2017; 34(3):213.

[41] Özdemir RBÖ, Özdemir AT, Sarıboyacı AE, Uysal O, Tuğlu Mİ, Kırmaz C. The Investigation of Immunomodulatory Effects of Adipose Tissue Mesenchymal Stem Cell Educated Macrophages on The CD4 T Cells. Immunobiology, 2019; 224(4):585-594.

[42] Wade RJ, Burdick JA. Engineering ECM Signals into Biomaterials. Materials Today, 2012; 15(10):454-459.

[43] Choi CK, Breckenridge MT, Chen CS. Engineered Materials and The Cellular Microenvironment: A Strengthening Interface Between Cell Biology and Bioengineering. Trends in Cell Biology, 2010; 20(12):705-714.

[44] Xiao S, Zhao T, Wang J et al. Gelatin methacrylate (GelMA)-Based Hydrogels for Cell Transplantation: An Effective Strategy for Tissue Engineering. Stem Cell Reviews and Reports, 2019; 15(5):664-679.

[45] Winer JP, Janmey PA, McCormick ME, Funaki M. Bone Marrow-Derived Human Mesenchymal Stem Cells Become Quiescent on Soft Substrates But Remain Responsive to Chemical or Mechanical Stimuli. Tissue Engineering Part A, 2009; 15(1):147-154.

[46] Dhall A, Lab C. Simulating Fluid Flow Through a Culture Chip for Cell Migration Studies in Microgravity. COMSOL Conference; 2016, Boston, USA.

[47] Song MJ, Brady-Kalnay SM, McBride SH, Phillips-Mason P, Dean D, Knothe Tate ML. Mapping The Mechanome of Live Stem Cells using A Novel Method to Measure Local Strain Fields in Situ at the Fluid-Cell Interface. PLoS One, 2012; 7(9):e43601.

[48] Song MJ, Dean D, Knothe Tate ML. In Situ Spatiotemporal Mapping of Flow Fields Around Seeded Stem Cells at The Subcellular Length Scale. PLoS one, 2010; 5(9):e12796.

[49] McBride SH, Falls T, Knothe Tate ML. Modulation of stem cell shape and fate B: Mechanical Modulation of Cell Shape and Gene Expression. Tissue Engineering Part A, 2008; 14(9):15731580 .

[50] Tate MLK, Falls TD, McBride SH, Atit R, Knothe UR. Mechanical Modulation of Osteochondroprogenitor Cell Fate. The International Journal of Biochemistry \& Cell Biology, 2008; 40(12):2720-2738. 